\title{
MATHEMATICAL ANALYSIS OF SOLUTE TRANSPORT EXPONENTIALLY VARIES WITH TIME IN UNSATURATED SOIL MEDIA
}

\author{
Raji J' ${ }^{1}$, Ramesh T. ${ }^{2}$, Venu Prasad K.K. ${ }^{3}$, S.R. Sudheendra ${ }^{4}$ \\ ${ }^{I}$ Department of Mathematics, T. John Institute of Technology, Bangalore, India. \\ rajijnathan@gmail.com \\ ${ }^{2}$ Department of Mathematics, Cambridge Institute of Technology, Bangalore,India. \\ rameshgollahalli@gmail.com \\ ${ }^{3}$ Department of Mathematics Govt. First Grade College Hethur, Sakleshpur (tq) India \\ kkvpmaths@gmail.com \\ ${ }^{4}$ Department of Mathematics, T. John Institute of Technology, Bangalore, India. \\ sudhanvasrs@yahoo.com
}

\begin{abstract}
The wide range of contamination sources is one of many factors contributing to the complexity of groundwater quality assessment. Contaminants containing different chemicals will pass through different hydrologic zones as they migrate through the soil to the water table. Mathematical analysis is presented for simultaneous dispersion and adsorption of a solute within homogenous and isotropic porous media in steady unidirectional flow fields. The dispersion systems are adsorbing the solute at rates proportional to their concentration and are subject to input concentrations that vary exponentially with time. In this study, the advection-dispersion equation has been solved analytically to evaluate the transport of pollutants which takes into account of distribution coefficient and porosity by considering input concentrations of pollutants. The solution is obtained using Laplace transform, moving coordinates and Duhamel's theorem is used to get the solution in terms of complementary error function. Mathematical solutions are developed for predicting the concentration of contaminants in adsorbing porous media for prescribed media and fluid parameters.
\end{abstract}

Keywords: Advection, Dispersion, Adsorption, Integral Transforms, Fick's Law, Moving Coordinates, Duhamel's Theorem

\section{MATHEMATICAL MODEL}

The Advection-Dispersion equation along with initial condition and boundary conditions can be written as

$\frac{\partial C}{\partial t}=D \frac{\partial^{2} C}{\partial z^{2}}-w \frac{\partial C}{\partial z}-\left(\frac{1-n}{n}\right) K_{d} C$

Initially, saturated flow of fluid of concentration, $C=0$, takes place in the porous media. At $t=0$, the concentration of the upper surface is instantaneously changed to $C=C_{0}$. Thus, the appropriate boundary conditions for the given model

$$
\left.\begin{array}{cc}
C(z, 0)=0 & z \geq 0 \\
C(0, t)=C_{0} e^{-\gamma t} & t \geq 0 \\
C(\infty, t)=0 & t \geq 0
\end{array}\right\} .
$$

The problem then is to characterize the concentration as a function of $\mathrm{z}$ and $t$.

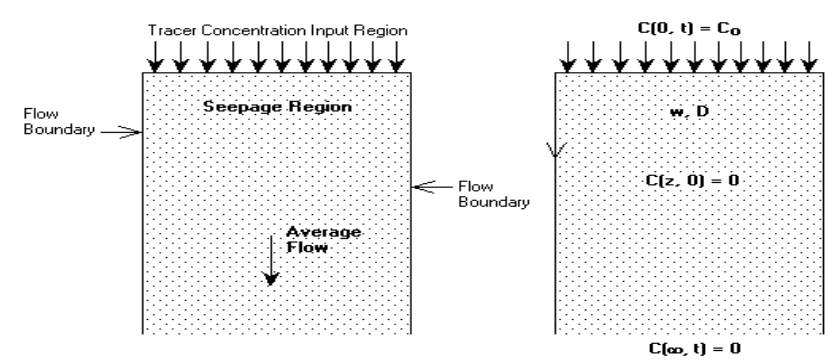

Figure 1 : Physical Layout of the Model

where the input condition is assumed at the origin and a second type or flux type homogeneous condition is assumed. $C_{0}$ is initial concentration. To reduce equation (3) to a more familiar form, we take

$C(z, t)=\Gamma(z, t) \operatorname{Exp}\left[\frac{w z}{2 D}-\frac{w^{2} t}{4 D}-\frac{K_{d}(1-n) t}{n}\right]$

Substituting equation ( 3 ) into equation (1) gives

$\frac{\partial \Gamma}{\partial t}=D \frac{\partial^{2} \Gamma}{\partial z^{2}}$ 
The initial and boundary conditions (2) transform to

$$
\left.\begin{array}{cc}
\Gamma(0, t)=C_{0} \operatorname{Exp}\left[\frac{w^{2} t}{4 D}+\frac{K_{d}(1-n) t}{n}-\gamma t\right] & t \geq 0 \\
\Gamma(z, 0)=0 & z \geq 0 \\
\Gamma(\infty, t)=0 & t \geq 0
\end{array}\right\}
$$

Equation (4) may be solved for a time dependent influx of the fluid at $z=0$. The solution of equation (4) may be obtained readily by use of Duhamel's theorem (Carslaw and Jaeger, 1947).

If $C=F(x, y, z, t)$ is the solution of the diffusion equation for semi-infinite media in which the initial concentration is zero and its surface is maintained at concentration unity, then the solution of the problem in which the surface is maintained at temperature $\phi(t)$ is

$$
C=\int_{0}^{t} \phi(\tau) \frac{\partial}{\partial t} F(x, y, z, t-\tau) d \tau
$$

This theorem is used principally for heat conduction problems, but the above has been specialized to fit this specific case of interest. Consider now the problem in which initial concentration is zero and the boundary is maintained at concentration unity. The boundary conditions are

$$
\left.\begin{array}{ll}
\Gamma(0, t)=0 & t \geq 0 \\
\Gamma(z, 0)=1 & z \geq 0 \\
\Gamma(\infty, t)=0 & t \geq 0
\end{array}\right\}
$$

The Laplace transform of equation (4) is

$$
L\left[\frac{\partial \Gamma}{\partial t}\right]=D \frac{\partial^{2} \Gamma}{\partial z^{2}}
$$

Hence, it is reduced to an ordinary differential equation

$$
\frac{\partial^{2} \bar{\Gamma}}{\partial z^{2}}=\frac{p}{D} \bar{\Gamma}
$$

The solution of the equation is $\bar{\Gamma}=A e^{-q z}+B e^{q z}$ where, $q= \pm \sqrt{\frac{p}{D}}$.

The boundary condition as $z \rightarrow \infty$ requires that $B=0$ and boundary condition at $z=0$ requires that $A=\frac{1}{p}$ thus the particular solution of the Laplace transformed equation is $\bar{\Gamma}=\frac{1}{p} e^{-q z}$

The inversion of the above function is given in any table of Laplace transforms. The result is

$$
\Gamma=1-\operatorname{erf}\left(\frac{z}{2 \sqrt{D t}}\right)=\frac{2}{\sqrt{\pi}} \int_{\frac{z}{2 \sqrt{D t}}}^{\infty} e^{-\eta^{2}} d \eta
$$

Using Duhamel's theorem, the solution of the problem with initial concentration zero and the time dependent surface condition at $z=0$ is

$\Gamma=\int_{0}^{t} \phi(\tau) \frac{\partial}{\partial t}\left[\frac{2}{\sqrt{\pi}} \int_{\frac{z}{2 \sqrt{D(t-\tau)}}}^{\infty} e^{-\eta^{2}} d \eta\right] d \tau$

Since $e^{-\eta^{2}}$ is a continuous function, it is possible to differentiate under the integral, which gives

$$
\frac{2}{\sqrt{\pi}} \frac{\partial}{\partial t} \int_{\frac{z}{2 \sqrt{D(t-\tau)}}}^{\infty} e^{-\eta^{2}} d \eta=\frac{z}{2 \sqrt{\pi D}(t-\tau)^{3 / 2}} \operatorname{Exp}\left[\frac{-z^{2}}{4 D(t-\tau)}\right]
$$

The solution to the problem is

$$
\Gamma=\frac{z}{2 \sqrt{\pi D}} \int_{0}^{t} \phi(\tau) \operatorname{Exp}\left[\frac{-z^{2}}{4 D(t-\tau)}\right] \frac{d \tau}{(t-\tau)^{3 / 2}}
$$

Putting $\mu=\frac{z}{2 \sqrt{D(t-\tau)}}$ then the equation (7) can be written as

$$
\Gamma=\frac{2}{\sqrt{\pi}} \int_{\frac{z}{2 \sqrt{D t}}}^{\infty} \phi\left(t-\frac{z^{2}}{4 D \mu^{2}}\right) e^{-\mu^{2}} d \mu
$$

Since $\phi(t)=C_{0} \operatorname{Exp}\left(\frac{w^{2} t}{4 D}+\frac{K_{d}(1-n) t}{n}-\gamma\right) \quad$ the particular solution of the problem may be written as

$$
\begin{aligned}
& \Gamma(z, t)=\frac{2 C_{0}}{\sqrt{\pi}} \operatorname{Exp}\left(\frac{w^{2} t}{4 D}+\frac{K_{d}(1-n) t}{n}-\gamma t\right) \\
& \left\{\int_{0}^{\infty} \operatorname{Exp}\left(-\mu^{2}-\frac{\varepsilon^{2}}{\mu^{2}}\right) d \mu-\int_{0}^{\alpha} \operatorname{Exp}\left(-\mu^{2}-\frac{\varepsilon^{2}}{\mu^{2}}\right) d \mu\right\}
\end{aligned}
$$

where, $\alpha=\frac{z}{2 \sqrt{D t}}$ and

$$
\varepsilon=\sqrt{\left(\frac{w^{2}}{4 D}+\frac{K_{d}(1-n)}{n}-\gamma\right)}\left(\frac{z}{2 \sqrt{D}}\right) \text {. }
$$

\section{EVALUATION OF THE INTEGRAL SOLUTION}

The integration of the first term of equation (9) gives 
$\int_{0}^{\infty} \operatorname{Exp}\left(-\mu^{2}-\frac{\varepsilon^{2}}{\mu^{2}}\right) d \mu=\frac{\sqrt{\pi}}{2} e^{-2 \varepsilon}$.

For convenience the second integral may be expressed on terms of error function (Horenstein, 1945), because this function is well tabulated.

Noting that

$-\mu^{2}-\frac{\varepsilon^{2}}{\mu^{2}}=-\left(\mu+\frac{\varepsilon}{\mu}\right)^{2}+2 \varepsilon=-\left(\mu-\frac{\varepsilon}{\mu}\right)^{2}-2 \varepsilon$.

The second integral of equation (9) may be written as

$$
\begin{aligned}
& I=\int_{0}^{\alpha} \operatorname{Exp}\left(-\mu^{2}-\frac{\varepsilon^{2}}{\mu^{2}}\right) d \mu \\
& =\frac{1}{2}\left\{e^{2 \varepsilon} \int_{0}^{\alpha} \operatorname{Exp}\left[-\left(\mu+\frac{\varepsilon}{\mu}\right)^{2}\right] d \mu+e^{-2 \varepsilon} \int_{0}^{\alpha} \operatorname{Exp}\left[-\left(\mu-\frac{\varepsilon}{\mu}\right)^{2}\right] d \mu\right\}
\end{aligned}
$$

Since the method of reducing integral to a tabulated function is the same for both integrals in the right side of equation (11), only the first term is considered. Let $a=\varepsilon / \mu$ and the integral may be expressed as

$$
\begin{aligned}
& I_{1}=e^{2 \varepsilon} \int_{0}^{\alpha} \operatorname{Exp}\left[-\left(\mu+\frac{\varepsilon}{\mu}\right)^{2}\right] d \mu \\
& =-e^{2 \varepsilon} \int_{\varepsilon / \alpha}^{\infty}\left(1-\frac{\varepsilon}{a^{2}}\right) \operatorname{Exp}\left[-\left(\frac{\varepsilon}{a}+a\right)^{2}\right] d a+ \\
& e^{2 \varepsilon} \int_{\varepsilon / \alpha}^{\infty} \operatorname{Exp}\left[-\left(\frac{\varepsilon}{a}+a\right)^{2}\right] d a
\end{aligned}
$$

Further, let, $\beta=\left(\frac{\varepsilon}{a}+a\right)$

in the $\beta=\frac{\varepsilon}{a}+a$ first term of the above equation, then

$$
I_{1}=-e^{2 \varepsilon} \int_{\alpha+\frac{\varepsilon}{\alpha}}^{\infty} e^{-\beta^{2}} d \beta+e^{2 \varepsilon} \int_{\frac{\varepsilon}{\alpha}}^{\infty} \operatorname{Exp}\left[-\left(\frac{\varepsilon}{a}+a\right)^{2}\right] d a
$$

Similar evaluation of the second integral of equation (11) gives

$$
\begin{aligned}
& I_{2}=e^{-2 \varepsilon} \int_{\varepsilon / \alpha}^{\infty} \operatorname{Exp}\left[-\left(\frac{\varepsilon}{a}-a\right)^{2}\right] d a- \\
& e^{-2 \varepsilon} \int_{\varepsilon / \alpha}^{\infty} \operatorname{Exp}\left[-\left(\frac{\varepsilon}{a}-a\right)^{2}\right] d a
\end{aligned}
$$

Again substituting $-\beta=\frac{\varepsilon}{a}-a$ into the first term, the result is

$$
I_{2}=e^{-2 \varepsilon} \int_{\frac{\varepsilon}{\alpha}-\alpha}^{\infty} e^{-\beta^{2}} d \beta-e^{-2 \varepsilon} \int_{\varepsilon / \alpha}^{\infty} \operatorname{Exp}\left[-\left(\frac{\varepsilon}{a}-a\right)^{2}\right] d a .
$$

Noting that

$$
\begin{aligned}
& \int_{\varepsilon / \alpha}^{\infty} \operatorname{Exp}\left[-\left(a+\frac{\varepsilon}{a}\right)^{2}+2 \varepsilon\right] d a= \\
& \int_{\varepsilon / \alpha}^{\infty} \operatorname{Exp}\left[-\left(\frac{\varepsilon}{a}-a\right)^{2}-2 \varepsilon\right] d a
\end{aligned}
$$

Substitution into equation (11) gives

$$
I=\frac{1}{2}\left(e^{-2 \varepsilon} \int_{\frac{\varepsilon}{\alpha}-\alpha}^{\infty} e^{-\beta^{2}} d \beta-e^{2 \varepsilon} \int_{\alpha+\frac{\varepsilon}{\alpha}}^{\infty} e^{-\beta^{2}} d \beta\right)
$$

Thus, equation (9) may be expressed as

$$
\begin{aligned}
& \Gamma(z, t)=\frac{2 C_{0}}{\sqrt{\pi}} \operatorname{Exp}\left(\frac{w^{2} t}{4 D}+\frac{K_{d}(1-n) t}{n}-\gamma\right) . \\
& \left\{\frac{\sqrt{\pi}}{2} e^{-2 \varepsilon}-\frac{1}{2}\left[e^{-2 \varepsilon} \int_{\frac{\varepsilon}{\alpha}-\alpha}^{\infty} e^{-\beta^{2}} d \beta-e^{2 \varepsilon} \int_{\alpha+\frac{\varepsilon}{\alpha}}^{\infty} e^{-\beta^{2}} d \beta\right]\right.
\end{aligned}
$$

However, by definition,

$$
e^{2 \varepsilon} \int_{\alpha+\frac{\varepsilon}{\alpha}}^{\infty} e^{-\beta^{2}} d \beta=\frac{\sqrt{\pi}}{2} e^{2 \varepsilon} \operatorname{erfc}\left(\alpha+\frac{\varepsilon}{\alpha}\right)
$$

also,

$$
e^{-2 \varepsilon} \int_{\frac{\varepsilon}{\alpha}-\alpha}^{\infty} e^{-\beta^{2}} d \beta=\frac{\sqrt{\pi}}{2} e^{-2 \varepsilon}\left(1+\operatorname{erf}\left(\alpha-\frac{\varepsilon}{\alpha}\right)\right) \text {. }
$$

Writing equation (15) in terms of error functions, we get

$$
\begin{aligned}
& \Gamma(z, t)=\frac{C_{0}}{2} \operatorname{Exp}\left(\frac{w^{2} t}{4 D}+\frac{K_{d}(1-n) t}{n}-\gamma t\right) \\
& {\left[e^{2 \varepsilon} \operatorname{erfc}\left(\alpha+\frac{\varepsilon}{\alpha}\right)+e^{-2 \varepsilon} \operatorname{erfc}\left(\alpha-\frac{\varepsilon}{\alpha}\right)\right]}
\end{aligned}
$$




$$
\begin{aligned}
& \frac{C}{C_{0}}=\frac{1}{2} \operatorname{Exp}\left[\frac{w z}{2 D}-\gamma\right] . \\
& {\left[e^{-2 \varepsilon} \operatorname{erfc}\left(\alpha-\frac{\varepsilon}{\alpha}\right)+e^{2 \varepsilon} \operatorname{erfc}\left(\alpha+\frac{\varepsilon}{\alpha}\right)\right]}
\end{aligned}
$$

Re-substituting for $\varepsilon$ and $\alpha$ gives

$$
\begin{aligned}
& \frac{C}{C_{0}}=\frac{1}{2} \operatorname{Exp}\left[\frac{w z}{2 D}-\gamma\right] \\
& {\left[\operatorname{Exp}\left[\frac{\sqrt{w^{2} n+4 D(1-n) K_{d}-4 D n \gamma}}{2 D \sqrt{n}} z\right]\right.} \\
& \operatorname{erfc}\left[\frac{z+\sqrt{w^{2} n+4 D(1-n) K_{d}-4 D n \gamma}}{2 \sqrt{D n t}} t\right]+ \\
& \operatorname{Exp}\left[-\frac{\sqrt{u^{2} n+4 D(1-n) K_{d}-4 D n \gamma}}{2 D \sqrt{n}} z\right. \\
& \operatorname{erfc}\left[\frac{z-\sqrt{u^{2} n+4 D(1-n) K_{d}-4 D n \gamma}}{2 \sqrt{D n t}}\right]
\end{aligned}
$$

where boundaries are symmetrical the solution of the problem is given by the first term the equation (17). The second term is equation (17) is thus due to the asymmetric boundary imposed in the more general problem. However, it should be noted also that if a point a great distance away from the source is considered, then it is possible to approximate the boundary condition by $C(-\infty, t)=C_{0}$, which leads to a symmetrical solution.

\section{RESULTS \& DISCUSSIONS:}

The main limitations of the analytical methods are that the applicability is for relatively simple problems. The geometry of the problem should be regular. The properties of the soil in the region considered must be homogeneous in the sub region. The analytical method is somewhat more flexible than the standard form of other methods for onedimensional transport model. Figures 1 to 4 represents the concentration profiles verses distance along the media for different values of porosity $\mathrm{n}$. It is seen that for a fixed velocity $\mathrm{w}$, dispersion coefficient $\mathrm{D}$ and distribution coefficient $\mathrm{K}_{\mathrm{d}}, \mathrm{C} / \mathrm{C}_{0}$ decreases with depth as porosity $\mathrm{n}$ decreases due to the distributive coefficient $\mathrm{K}_{\mathrm{d}}$, whereas concentration profile versus time for different values of depth $\mathrm{z}$. For a fixed $\mathrm{z}$ it is seen that concentration increases in the beginning due to lesser effect of dispersion coefficient $\mathrm{D}$ and reaches a steady-state value for larger time.

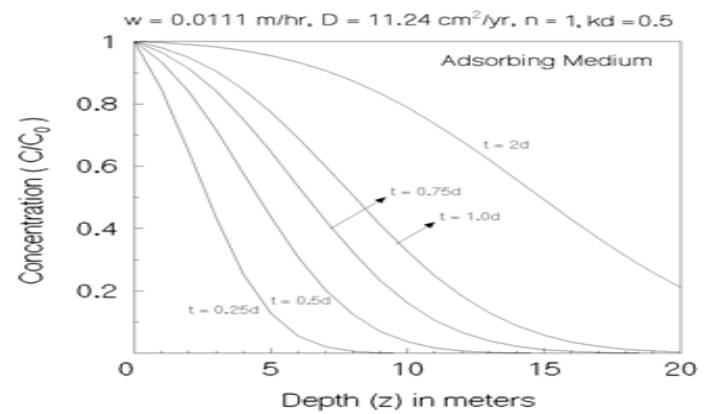

Fig. 1: Break-through-curve for $\mathrm{C} / \mathrm{C}_{0} \mathrm{v} / \mathrm{s}$ depth for $\mathrm{n}=1.0, \mathrm{~K}_{\mathrm{d}}=0.5 \& \gamma=0$

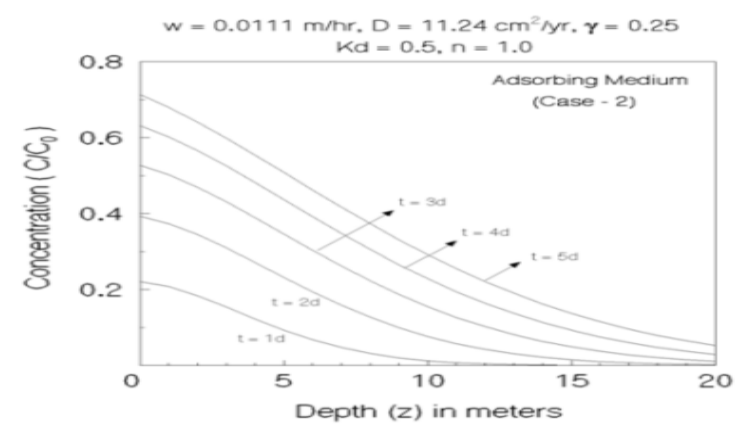

Fig. 2: Break-through-curve for $\mathrm{C} / \mathrm{C}_{0} \mathrm{v} / \mathrm{s}$ depth for $n=1.0, K_{d}=0.5 \& \gamma=0.25$

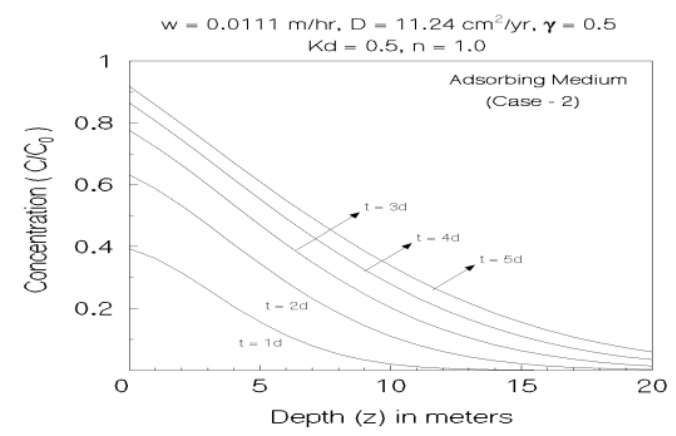

Fig. 3: Break-through-curve for $\mathrm{C} / \mathrm{C}_{0} \mathrm{v} / \mathrm{s}$ depth for $\mathrm{n}=1.0, \mathrm{~K}_{\mathrm{d}}=0.5 \& \gamma=0.5$

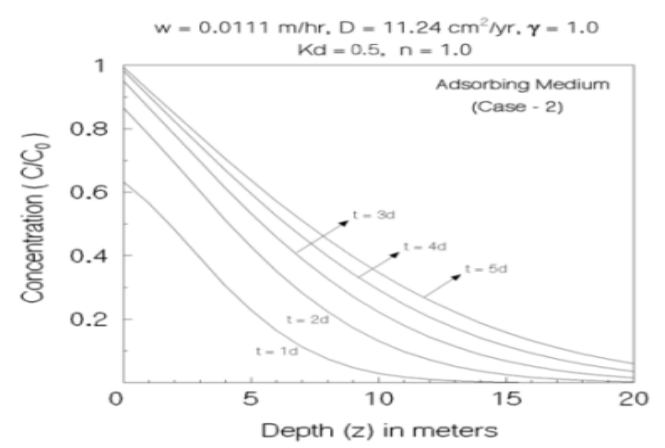

Fig. 4: Break-through-curve for $\mathrm{C} / \mathrm{C}_{0} \mathrm{v} / \mathrm{s}$ depth for $\mathrm{n}=1.0$,

$$
\mathrm{K}_{\mathrm{d}}=0.5 \& \gamma=1.0
$$

The figures represent $C / C_{0}$ verses time for different values of distribution coefficient $K_{d}$. It is seen that for a fixed $K_{d}$, concentration increases slowly up to $\mathrm{t}=10$ days because of the less adsorption of pollutants on the solid surface and then reaches a constant value for larger time where the effect of distribution coefficient $\mathrm{K}_{\mathrm{d}}$ is small. We conclude 
that the integral transform method is a powerful method to derive analytical solutions for solute transport of a adsorption in homogeneous porous media and under different flow conditions. Steady-state concentration distributions and temporal moments can be directly derived from these solutions and transient concentration distribution is accessible via numerical inversion. The derived solutions are of great value for bench-marking numerical reactive transport codes.

\section{REFERENCES:}

[1]. Aral,M.M., Liao, B., 1996. Analytical solutions for two - dimensional transport equation with time-dependant dispersion co-efficient. Journal of Hydrologic Engineering, 1,20-32.

[2]. Barry,D.A.,and Sporito,G.,1989. Analytical solution of a convection-dispersion model with time-dependant transport co-efficients. Water Resour.Res.,25,24072416.

[3]. Batu,V.,1993. A genaralized two-dimensional analytical solute transport model in bounded media for flux-type multiple sources. Water Resour.Res.,29,2881-2892.

[4]. Bear,J.,and A.Verruijt.,1990.Modelling Groundwater flow and pollution.D Radial Publishing Co.,Tokyo.

[5]. Ermak,D.L.,1977.An Analytical Model for Air Pollutant transport and deposition from a point source.Atmos.Environ., 11,231-237.

[6]. J.S.Chen,C.W.Liu, and C.M.Liao.,2003.Twodimensional Laplace-Transformed Power Series Solution for Solute Transport in a Radially Convergent Flow Field.Adv.Water Res.,26,1113-1124.

[7]. Koch,W.,1989.A Solution of two-dimensional atmosphere diffusion equation with height-dependent diffusion coefficient including ground level absorption. Atmos. Environ., 23, 1729-1732.

[8]. Sudheendra S.R., 2010 A solution of the differential equation of longitudinal dispersion with variable coefficients in a finite domain, Int. J. of Applied Mathematics \& Physics, Vol.2, No. 2, 193-204.

[9]. Sudheendra S.R., 2011. A solution of the differential equation of dependent dispersion along uniform and non-uniform flow with variable coefficients in a finite domain, Int. J. of Mathematical Analysis, Vol.3, No. 2, 89-105.

[10].Sudheendra S.R. 2012. An analytical solution of onedimensional advection-diffusion equation in a porous media in presence of radioactive decay, Global Journal of Pure and Applied Mathematics, Vol.8, No. 2, 113124.

[11].Sudheendra S.R., Raji J, \& Niranjan CM, 2014. Mathematical Solutions of transport of pollutants through unsaturated porous media with adsorption in a finite domain, Int. J. of Combined Research \& Development, Vol. 2, No. 2, 32-40.

[12].Sudheendra S.R., Praveen Kumar M. \& Ramesh T. 2014. Mathematical Analysis of transport of pollutants through unsaturated porous media with adsorption and radioactive decay, Int. J. of Combined Research \& Development, Vol. 2, No. 4, 01-08.
[13].Sudheendra S.R., Raji J, \& Niranjan CM, 2014. Mathematical modelling of transport of pollutants in unsaturated porous media with radioactive decay and comparison with soil column experiment, Int. Scientific J. on Engineering \& Technology, Vol. 17, No. 5.

[14].Tartakowsky,D.,Di Federico,V.,1997. An analytical solution for contaminant transport in non-uniform flow. Transport in porous media, 27, 85-97.

[15].Wexler.E.J.,1992.Analytical solution for one, two and three dimensional solute transport in Ground water systems with uniform flow. U.S. Geological Survey, Techniques of water Resources Investigations, Book 3,Chap. B.7.

[16].Yates,S.R.,1990. An analytical solution for onedimensional transport in heterogeneous porous media. Water Resour. Res., 26, 2331-2338.

[17].Zoppou,C., and Knight, J.H..,1997.Analytical solution for advection and advection-diffusion equation with spatially variable coefficients. Journal of Hydraulic Engineering., 123,144-148. 